\title{
Über die Existenz von Isotopen der Actiniumzerfallsprodukte und das Meitnersche Kernmodell.
}

Von Maximilitan Camillo Neuburger.

Die Actiniumfamilie weist gegenüber der Radium- und Thoriumfamilie Mängel an Analogie auf, welche Unstimmigkeiten bei den Beziehungen zwischen Atomgewicht und Lebensdauer, bzw. Zerfallswahrscheinlichkeit und Reichweite hervorrufen. Diese Analogiemängel $\left.{ }^{1}\right)$ sind die folgenden:

1. Die eine der beiden bei Radioactinium experimentell bestimmten Reichweiten $(4,61$ and $4,2 \mathrm{~cm})$ ist größer wie die von Actinium $X$ (Reichweite $4,26 \mathrm{~cm}$ ), obwohl sonst immer größeren Reichweiten kürzere Lebensdauern zugeordnet sind.

2. Der $\alpha$-Strahler Radioactinium emittiert, eine $\beta$-Strahlung $(v / c=0,37,0,39,0,43,0,50,0,57,0,60,0,66)$, welcher derjenigen von Mesothorium 2 sehr ähnlich ist.

3. Radioactinium emittiert eine $\gamma$-Strahlung die der von Mesothorium ( $\mu$ in $\mathrm{Al}=26$ und 0,116 für MsTh gegen $\mu=25$ und 0,19 für RdAc) sehr nahe steht. Im Gegensatz hierzu ist bei Radiothorium keine solche Strahlung gefunden worden.

4. Nach E. RUturerford und H. Richardson soll Actinium eine $\gamma$-Strahlung anssenden, deren Intensität ca. $25 \%$ der Gesamt- $\gamma$ Strahlung ron Actinium samt allen Folgeprodukten im radioaktiven Gleichgewicht beträgt.

5. Die Fajanssche Beziehung zwischen Atomgewicht und Lebensdauer von Isotopen versagt bei Actinium $X$ und Actinium $B$.

6. Die Analogie zur Thoriumreihe fehlt gänzlich im Gebiete der Mesothorium-Radiothoriumprodukte.

STEFAn MEYeR ${ }^{1}$ ) hat Versuche zwecks Überprüfung dieser Analogiemängel ausgeführt und auBerdem noch speziell die Frage experimentell zu lösen gesucht, ob diese UnregelmäBigkeiten etwa

1) St. MeqER, Mitteilung aus dem Institut für Radiumforschung Nr. 104 und $Z$. phys. Chem. 95 (1920), 407-433, Nr. 4. 
durch Existenz ron unbekannten Isotopen zu den Umwandlungsprodukten der Actiniumfamilie verursacht werden.

Die folgenden Umwandlungsschematen könnten die angeführten Unstimmigkeiten erklären. ${ }^{1}$ )

1.

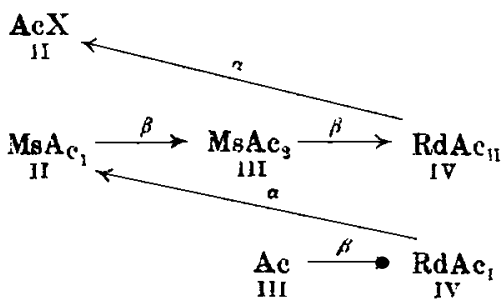

verlangt Isotope za Ac, BdAc und AeX

2.

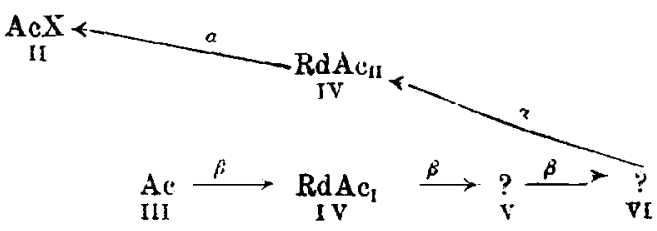

3.

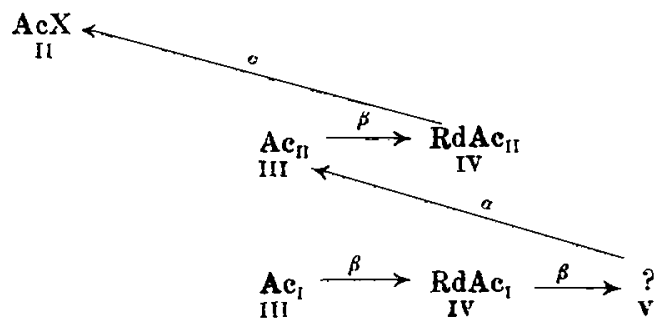

4.
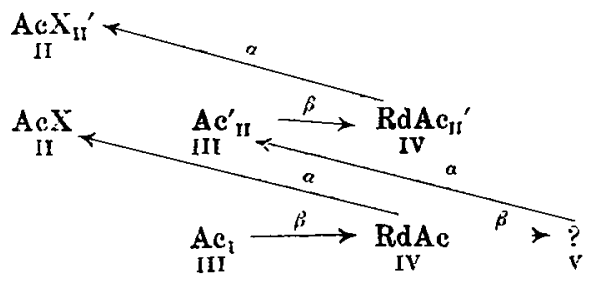

5.

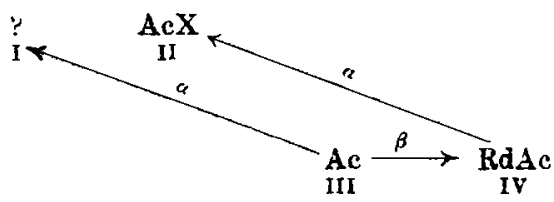

fordert je ein Isutop zu RdAc, Pa und $\mathrm{U}$

fordert Isotope zu Ac, RdAc und $\mathrm{Pa}$ fordert Isotope zu Ac, RdAc, AcX und $\mathrm{Pa}$. fordert das unbekannte radioaktive Alkalimetall, könnte jedoch die Unstimmigkeiten nicht erklären.

1) Siehe auch die demnächst erscheinende Monographie: M. C. NEvberger, „Das Problem des Genesis des Actiniums". Stuttgart, Verlag von Ferd. Enke. 
Die Versuche, diese Isotopen aufzufinden, ergaben ausnahmslos ein negatives Resultat, so daB die Analogiemängel durch Isotope nicht hervorgerufen sein können. Das Ergebnis dieser Arbeiten von Stefan Meyer wird aber durch eine neue, vor kurzer Zeit von Lise Meitsen aufgestellte Hypothese über den Aufbau der Atomkerne von radioaktiven Elementen erklärt bzw. gefordert.

Nach Lise Mritner ${ }^{1}$ ) bestehen die Atomkerne derjenigen Radioelemente, deren Atomgewicht von der Form $4 \mathrm{n}$. ist, zur Gänze aus Heliumkernen, während Elemente, deren Atomgewicht den Formeln t $\mathrm{n} .+1,4 \mathrm{n} .+2,4 \mathrm{n}+3$ entspricht, aus Wasserstoffkernen und Heliumkernen aufgebaut sind (abgesehen von den Elektronen).

Die folgenden Buchstaben haben die angegebene Bedeutung:

A . . . Atomgewicht,

x..... Ordnungszahl,

n. . . G Gesamtzahl der Heliumkerne ( $\alpha$-Teilchen),

p. . . . Anzahl der Wasserstoffatome,

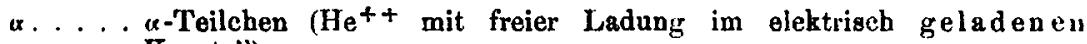
Kernteil),

$\alpha^{\prime} . \ldots \alpha$-Teilchen mit gebundener Ladung (im He-Atom bzw. He+.Ion), $\beta . .$. Elektron im He-Atom, bzw. $\mathrm{He}^{+}$-Ion,

$H^{+} \ldots$ Wasserstoff kern,

e..... Elektron im Wasserstofiatom,

$\left(\alpha^{\prime}+2 \beta\right)$ elektrisch neutraler Kernteil (He-Atom),

$N_{k}$. . . Gesamtsumme der Kernbausteine,

so ist das Atomgewicht irgendeines Elementes durch die Formel

$$
A=4 n+p \quad(p=0,1,2,3)
$$

bestimmt. Die Gesamtsumme der Kernbestandteile eines Atomkernes von gerader Ordnungszahl ist dann

$$
N_{k}=\frac{z}{2} \alpha+\left(n-\frac{z}{2}\right)\left(\alpha^{\prime}+2 \beta\right)+\mu\left(H^{+}+e\right) .
$$

Eine ungerade Kernladung kann nach diesem Kernmodell nur durch ein freies überschilissiges, aus dem neutralen Kernteil stammendes Elektron - $(\boldsymbol{\beta})$ - verursacht werden.

Bei Instabilität eines nach obiger Formel zusammengesetzten Atomkernes kann entweder $\alpha$ - oder $\beta$ Strahlung emittiert werden. Die $\alpha$-Strahlung kann aus dem elektrisch geladenen oder aus dem elektrisch neutralen Kernteil stammen. Beginnt der radioaktive

1) Lisi Meitner, Z. f. Physite 4 (1921), 146-156, Nr. 1, sowie Naturwissensehaften 9 (1921), 423-427, Nr. 22, und Festschrift der Kaiser-WilhelmGesellsehaft, S. 154. 
Zerfall mit der Emission von $\alpha$-Teilchen, so wird, da die Anzahl $\operatorname{der}\left(\alpha^{\prime}+2 \beta\right)$-Teilchen weit geringer ist, wahrscheinlich eine Reihe von $\alpha$-Umwandlungen folgen, bevor der elektrisch neutrale Kernteil instabil wird. Für weitere Einzelheiten mủssen wir auf die zitierten Originalarbeiten von Lise Mritwer verweisen. Nach diesem Kernmodell sind folgende Zerfallsschematen möglich:

1. „Eine Reihe aufeinander folgender $\alpha$-Umwandlungen":

$$
\alpha-\alpha-\alpha-\alpha \text {. }
$$

2. „Eine $\alpha$-Umwandlung der zwei $\beta$-Umwandlungen folgen":

bzw. auch

$$
\alpha^{\prime}-\beta-\beta
$$

und

$$
\beta-\alpha^{\prime}-\beta
$$

$$
\beta-\beta-\alpha^{\prime}
$$

3. „Eine $\beta$-Umwandlung, die zu einer Verzweigung fuhrt, wobei nach zwei Umwandlungsstufen die Zweige sich wieder schlieBen":<smiles>[3H][C@H]1C[C@H](C)C1</smiles>

4. „Eine Verzweigung der Form<smiles>CCCCC(C)C</smiles>

die durch Spaltung in zwei selbständige Reihen führt").

Was nun die Umwandlungsschematen 2., 3. und 4. betrifft, so ist zu bemerken, daB diesen Schematen die Annahme zugrunde gelegt ist, daB Radioactinium $\beta$-Umwandlungsprodukte bildet. OтTo HAHN ${ }^{1)}$ hat experimentell festgestellt, daB Radium und Radioactinium keine solchen Umwandlungsprodukte erzeugen.

Die für die Aktivität gefundenen Werte waren etwa $10000 \mathrm{mal}$ kleiner, als die $z u$ erwartenden. Man müBte danach annehmen - da mit der Emission von $\beta$-Strahlen keine Umwandlnng des Atoms einzutreten scheint -, daB sie nicht aus dem Kern kommen. Doch ist diese Annahme mit Schwierigkeiten verknüpft, da sie die Frage nach der Energiequelle dieser $\beta$-Teilchen nicht zu lösen vermag.

In dem Meitnerschen Kernmodell der Radioelemente ist das

1) Otro HAHN, Z. f. Physik 2 (1921), 60. 
experimentelle Ergebnis Oтто Haws s schon berücksichtigt. Schreibt man die Formel für die Summe der Kernbausteine an,

$$
\underline{N_{k}^{\mathrm{RdAc}}=45 \alpha+11\left(\alpha^{\prime}+2 \beta\right)+2\left(B^{+}+e\right),}
$$

so bemerkt man sofort, daB Radioactinium keine Kern- $\beta$-Strahlung emittieren kann, da sein Atomkern kein freies $\beta$-Elektron enthält. Nichtsdestoweniger könnte sich aber ein $\left(\alpha^{\prime}+2 \beta\right)$-Teilchen aufspalten und so die Emission von $\beta$-Teilchen verursachen. Die Wahrscheinlichkeit dieser Aufspaltung ist aber sehr gering, da dieser Fall erst dann leichter eintreten könnte, wenn viel weniger als 45 a-Teilchen im Kern des Radioactiniumatoms vorhanden sind. Um dies einzusehen, ist es nur nötig, die $N_{k}$-Formeln oder Radioelemente in genetischer Reihenfolge anzuschreiben.

\section{Uran - Radiumfamilie.}

$$
\begin{aligned}
& \mathrm{U}_{1} \ldots N_{k}=46 \alpha+13\left(\alpha^{\prime}+2 \beta\right)+2\left(H^{+}+e\right) \\
& \mathrm{UX}_{1} \ldots N_{k}=46 \alpha+12\left(\alpha^{\prime}+2 \beta\right)+2 \beta+2\left(H^{+}+e\right) \\
& \mathrm{UX}_{2} \ldots N_{k}=46 \alpha+12\left(\alpha^{\prime}+2 \beta\right)+\beta+2\left(H^{+}+e\right) \\
& \mathrm{U}_{\mathrm{II}} \ldots N_{k}=46 \alpha+12\left(\alpha^{\prime}+2 \beta\right)+2\left(H^{+}+e\right) \\
& \text { Jo.... } \left.N_{k}=45 \alpha+12\left(\alpha^{\prime}+2 \beta\right)+2 H^{+}+e\right) \\
& \mathrm{Ra} . . . N_{k}=44 \alpha+12\left(\alpha^{\prime}+2 \beta\right)+2\left(H^{+}+e\right) \\
& \text { RaEm. . } N_{k}=43 \alpha+12\left(\alpha^{\prime}+2 \beta\right)+2\left(H^{+}+e\right) \\
& \operatorname{RaA} \ldots N_{k}=42 \alpha+12\left(\alpha^{\prime}+2 \beta\right)+2\left(H^{+}+e\right) \\
& \operatorname{RaB} \ldots N_{k}=41 \alpha+12\left(\alpha^{\prime}+2 \beta\right)+2\left(H^{+}+e\right) \\
& \mathrm{RaC} . . N_{k}=41 \alpha+11\left(\alpha^{\prime}+2 \beta^{\prime}\right)+\left(\alpha^{\prime}+\beta\right)+2\left(H^{+}+e\right) \\
& \mathrm{RaC}^{\prime} . . N_{k}=41 \alpha+11\left(\alpha^{\prime}+2 \beta\right)+\alpha^{\prime}+2\left(H^{+}+e\right) \\
& \mathrm{RaC}^{\prime} \ldots N_{k}=41 \alpha+11\left(\alpha^{\prime}+2 \beta\right)+\beta+2\left(H^{+}+e\right) \\
& \mathrm{RaD} . . . N_{k}=41 \alpha+11\left(\alpha^{\prime}+2 \beta\right)+2\left(H^{+}+e\right) \\
& \operatorname{RaE} \ldots N_{k}=41 \alpha+10\left(\alpha^{\prime}+2 \beta\right)+\left(\alpha^{\prime}+\beta\right)+2\left(H^{+}+e\right) \\
& \text { RaF . . . N } N_{k}=41 \alpha+10\left(\alpha^{\prime}+2 \beta\right)+\alpha^{\prime}+2\left(H^{+}+e\right) \\
& \text { RaG. . . } N_{k}=41 \alpha+10\left(\alpha^{\prime}+2 \beta\right)+2\left(H^{+}+e\right)
\end{aligned}
$$

\section{Actiniumfamilie.}

$$
\begin{aligned}
& \mathrm{U}_{\mathrm{rI}} \ldots . N_{k}=46 \alpha+12\left(\alpha^{\prime}+2 \beta\right)+2\left(H^{+}+e\right) \\
& \mathrm{UY} \ldots N_{k}=46 \alpha+11\left(\alpha^{\prime}+2 \beta\right)+2 \beta+2\left(B^{+}+e\right) \\
& \text { Pa. . . . N } N_{k}=46 \alpha+11\left(\alpha^{\prime}+2 \beta\right)+\beta+2\left(H^{+}+e\right) \\
& \text { Ac. . . . } N_{k}=45 \alpha+11\left(\alpha^{\prime}+2 \beta\right)+\beta+2\left(H^{+}+e\right) \\
& \text { RdAc . . } N_{k}=45 \alpha+11\left(\alpha^{\prime}+2 \beta\right)+2\left(H^{+}+e\right) \\
& \text { AcX. . . } N_{k}=44 \alpha+11\left(\alpha^{\prime}+2 \beta\right)+2\left(H^{+}+e\right) \\
& \text { AcEm . . } N_{k}=43 \alpha+11\left(\alpha^{\prime}+2 \beta\right)+2\left(H^{+}+e\right) \\
& \text { AcA . . . N } N_{k}=42 \alpha+11\left(\alpha^{\prime}+2 \beta\right)+2\left(H^{+}+e\right) \\
& \text { AcB . . . } N_{k}=41 \alpha+11\left(\alpha^{\prime}+2 \beta\right)+2\left(H^{+}+e\right) \\
& \mathrm{AcC} \ldots N_{k}=41 \alpha+10\left(\alpha^{\prime}+2 \beta\right)+\left(\alpha^{\prime}+\beta\right)+2\left(H^{+}+e\right) \\
& \mathrm{AcC}^{\prime} . . N_{k}=41 \alpha+10\left(\alpha^{\prime}+2 \beta\right)+\alpha^{\prime}+2\left(H^{+}+e\right) \\
& \mathrm{AcC}^{\prime} . N_{k}=41 \alpha+10\left(\alpha^{\prime}+2 \beta\right)+\beta+2\left(H^{+}+e\right) \\
& \text { AcD . . . } N_{k i}=41 \alpha+10\left(\alpha^{\prime}+2 \beta\right)+2\left(H^{+}+e\right)
\end{aligned}
$$


Thoriumfamilie.

$$
\begin{aligned}
& \text { Th. . . . } N_{k}=45 \alpha+13\left(\alpha^{\prime}+2 \beta\right) \\
& \mathrm{MsTh}_{1} \ldots N_{k}=45 \alpha+12\left(\alpha^{\prime}+2 \beta\right)+2 \beta \\
& \mathrm{MBTh}_{\mathrm{z}} \ldots N_{k}=45 \alpha+12\left(\alpha^{\prime}+2 \beta\right)+\beta \\
& \mathrm{RdTh} \ldots N_{k}=45 \alpha+12\left(\alpha^{\prime}+2 \beta\right) \\
& \text { ThX. . . } N_{k}=44 \alpha+12\left(\alpha^{\prime}+2 \beta\right) \\
& \text { ThEm . . } N_{k}=43 \alpha+12\left(\alpha^{\prime}+2 \beta\right) \\
& \text { ThA. . . } N_{k}=42 \alpha+12\left(\alpha^{\prime}+2 \beta\right) \\
& \text { ThB. . . } N_{k}=41 \alpha+12\left(\alpha^{\prime}+2 \beta\right) \\
& \text { ThC. . . } N_{k}=41 \alpha+11\left(\alpha^{\prime}+2 \beta\right)+\left(\alpha^{\prime}+\beta\right) \\
& \text { ThC'. . . } N_{k}=41 \alpha+11\left(\alpha^{\prime}+2 \beta\right)+\alpha^{\prime} \\
& \mathrm{ThC}^{\prime \prime} . . N_{k}=41 \alpha+11\left(\alpha^{\prime}+2 \beta\right)+\beta \\
& \text { ThD. . . } N_{k}=41 \alpha+11\left(\alpha^{\prime}+2 \beta\right)
\end{aligned}
$$

Da das als $\alpha$ - bezeichnete $\alpha$-Teilchen aus dem neutralen Kernteil - wie die Kernformeln von Radium $C^{\prime}$, Radium $F$, Actinium $C^{\prime}$ und Thorium $C^{\prime}$ zeigen - durch die zuerst erfolgte Emission der beiden $\beta$-Teilchen zu einem $\alpha$-Teilchen mit freier Kernladung wird, kann dieses $\alpha^{\prime}$-Teilchen als $\alpha$-Teilchen bezeichnet werden, da es mit letzterem vollkommen identisch ist. Die Kernformeln für Radium $C^{\prime}$, Radium $F$, Actinium $C^{\prime}$ und Thorium $C^{\prime}$ sind daher zu schreiben:

$$
\begin{aligned}
& \operatorname{RaC} \ldots N_{k}=42 \alpha+11\left(\alpha^{\prime}+2 \beta\right)+2\left(H^{+}+e\right) \\
& \operatorname{RaF} \ldots N_{k}=42 \alpha+10\left(\alpha^{\prime}+2 \beta\right)+2\left(H^{+}+e\right) \\
& \operatorname{AcC}^{\prime} \ldots N_{k}=42 \alpha+10\left(\alpha^{\prime}+2 \beta\right)+2\left(H^{+}+e\right) \\
& \operatorname{ThC}^{\prime} \ldots N_{k}=42 \alpha+11\left(\alpha^{\prime}+2 \beta\right)
\end{aligned}
$$

Die Umwandlungsschematen 2., 3., 4. sind also sowohl nach den experimentellen Ergebnissen von S'TFFan MEYER, als auch nach dem Meitwenschen Kernmodell unwahrscheinlich. Schema 5. ist ebenfalls za verwerfen, da Actinium kein $\alpha$-Strahler ist. Vorerst harrt jedoch noch die auffallende Tatsache, daB Radioactinium c-Teilchen verschiedener Reichweite emittiert, der Aufklärung. Die Emission von $\lll-T e i l c h e n$ der beiden Reichsweiten

bzw.

$$
R_{0}^{760} \ldots 4,37 \mathrm{~cm} \text { und } 4,0 \mathrm{~cm}
$$

$$
R_{15}^{760} \ldots 4,61 \mathrm{~cm} \text { und } 4,22 \mathrm{~cm}
$$

deutet auf eine Verzweigung der Actiniumfamilie bei Radioactinium hin. Da aber die Existenz von neuen Isotopen zu den Zerfallsprodukten der Actiniumfamilie nicht nachgewiesen werden konnte, können diese Isotopen nur dann der Auffindung entgangen sein, wenn das Verzweigungsverhältnis sehr klein ist. Der von A. SMrrraL berechnete Wert dieses Abzweigverhältnisses ist daher jedenfalls unrichtig, da der Wert von $1,7 \%$ viel zu hoch ist. Wir haben 
schon an anderer Stelle $^{1}$ ) nachgewiesen, daB die Methode, die SMreKaI zur Berechnung von Verzweigungsverhältnissen anwendet, ganz ungeeignet ist.

Das Umwandlungsschema 1. ist ebenfalls nach dem MeIrNesschen Kernmodell abzulehnen. Um den Zerfallsgang zu erklären, müBte man annehmen, daB ein $\left(\alpha^{\prime}+2 \beta\right)$-Teilchen aufbricht.

Die Kernformeln für Schema 1. wären:

$$
\begin{aligned}
& \operatorname{RdAc}_{1} \ldots N_{k}=45 \alpha+11\left(\alpha^{\prime}+2 \beta\right)+2\left(H^{+}+e\right) \\
& \mathrm{agAc}_{1} \ldots N_{k}=45 \alpha+10\left(\alpha^{\prime}+2 \beta\right)+2 \beta+2\left(H^{+}+e\right) \\
& \text { B I } \\
& { }_{\text {MsAc }}^{\beta} \ldots N_{i}=45 \alpha+10\left(\alpha^{\prime}+2 \beta\right)+\beta+2\left(H^{+}+e\right) \\
& \boldsymbol{\beta} \\
& { }_{\mathrm{RdAc}} \cdot N_{k}=45 \alpha+10\left(\alpha^{\prime}+2 \beta\right)+2\left(H^{+}+e\right) \\
& \text { a } \\
& \text { AcX. . . N } N_{k}=44 \alpha+10\left(\alpha^{\prime}+2 \beta\right)+2\left(H^{+}+e\right)
\end{aligned}
$$

Die Existenz der beiden $\beta$-Strahler Mesoactiniam 1 und Mesoactinium 2 könnte wohl nicht verborgen geblieben sein, da eine "strahlenlose" $\beta$-Umwandlung bei beiden Radioelementen nicht stattfinden kann. Schema 1. ist auch deshalb unwahrscheinlich, da für das Endprodukt der Actiniumfamilie nur das Atomgewicht 202 möglich wäre, was für Actinium $D$ nach den experimentellen Untersuchungen uber die Bleiisotopen ausgeschlossen ist.

Wir halten zwecks Erklärung der dualen $\alpha$-Emission von Radioactinium folgendes Umwandlungsschema für möglich $\left(\mathrm{AcEm}_{\mathrm{I}}\right.$ ist die bisher bekannte Actiniumemanation):

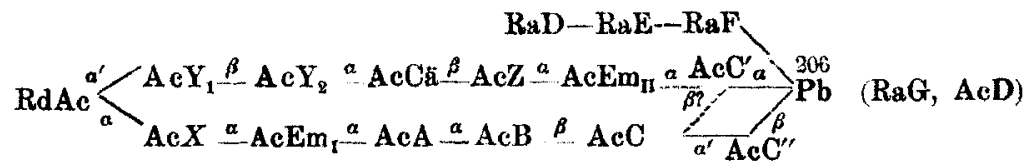

Wir haben oben bemerkt, daB die Wahrscheinlichkeit der Aufspaltung eines $\left(\alpha^{\prime}+\beta\right)$. Teilchens im Radioactiniumkern sehr gering ist; denn tatsächlich läßt sich diese Möglichkeit nicht völlig ausschließen. Das Verzweigungsverhältnis der beiden Zweigreihen kann daher auch nur sehr klein sein, $\mathbf{d} . \mathbf{h}$. höchstens wenige Promille betragen. Damit steht natürlich die Folgerung in Einklang, daB die neuen Isotopen der Actiniumzerfallsprodukte nur schwer nachweisbar sein werden. Es ist deshalb leicht möglich, daB sie sich der Auffindung bei den Versuchen von Stefias MEYer entzogen haben.

Bezüglich der in unserem Umwandlungsschema durchgeführten Vereinigung der Radium- und Actiniumfamilie in dem gemein-

1) M. C. Neunuraen, Z. phys. Chem. 99 (1921), 168-171; Nr. 3/4. 
schaftlichen Endprodukt Blei vom Atomgewicht 206 verweisen wir auf unsere Arbeit ${ }^{1}$ ) „Die Isotopie der Radioelemente und das Meitner sche Kernmodell".

Die Kernformeln der neuen Isotopen sind:

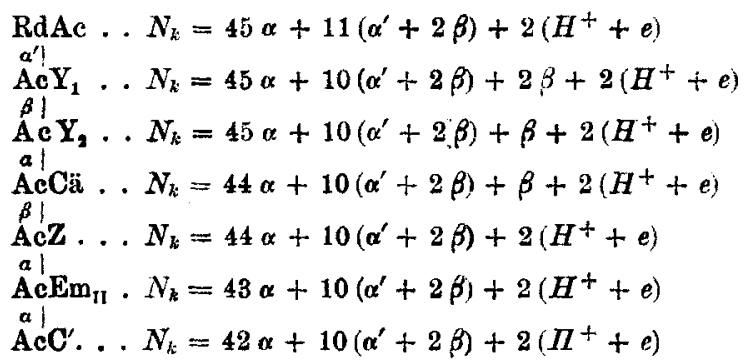

Das Actinium $C^{\prime}$ dieser Kernformeln muB mindestens ein Isotop dritter Ordnung ${ }^{1}$ ) des bekannten Actinium $C^{\prime}$ sein, oder auch ein Isotop vierter Ordnung dieses Radioelements. Es könnte jedoch auch mit dem bisher bekannten Actinium $C^{\prime}$ vollkommen identisch sein. Die letztere Annahme ist in unserem Umwandlungsschema zum Ausdruck gebracht worden.

Das Actinocäsium (AcCä) wäre das bisher vergeblich gesuchte radioaktive Alkalimetall der Ordnungszahl 87.

In unserem Umwandlungsschema, sowie in dem experimentell sichergestellten Zerfallsschema der Actiniumfamilie von Uran II bis Radioactinium ist der Zerfallsgang folgender

$$
\alpha^{\prime}-\beta-\alpha-\beta \text {. }
$$

Diesen Zerfallsgang bezeichnet Lise Meitnke als Ausnahme, da die in ihrer Arbeit gegebenen Zerfallsschematen bei Aufspaltung eines $\left(\alpha^{\prime}+2 \beta\right)$-Teilchens

$$
\begin{aligned}
& \alpha^{\prime}-\beta-\beta \\
& \beta-\alpha-\beta \\
& \beta-\beta-\alpha^{\prime}
\end{aligned}
$$

eine dazwischen eintretende $\propto$-Strahlung nicht berücksichtigen. Im Gegensatz zur Auffassung von LIse MEITNER möchten wir hervorheben, dab unseres Erachtens Zerfallsgang (I) keine Abweichung darstellt, da diese Umwandlungsfolge in dem Aufbau der Atomkerne der betreffenden Radioelemente insofern begründet ist, als die Zerfallswahrscheinlichkeit in der Richtung zum Uran $Y$ bei Uran II viel kleiner ist als zur Radiumfamilie, d.h. es besteht eine viel

1) M. C. Neuguragr, Z phys. Chem. 99 (1921), 161-167; Nr. 3/4. 
geringere Wahrscheinlichkeit für das Aufbrechen eines $\left(\alpha^{\prime}+2 \beta\right)$ Teilchens als für die Emission eines $\alpha$-Teilchens. Es überwiegt also die Instabilität des elektrisch geladenen Kernteils die des elektrisch neutralen Kernteils und es ist daher erklärlich, daB die durch Aufspaltung eines $\left(\alpha^{\prime}+2 \beta\right)$-Teilchens noch gesteigerte Instabilität des elektrisch geladenen Kernteils die Emission von $\alpha$-Teilchen bedingt, obwohl noch ein freies Elektron im Kern vorhanden ist.

Das hier gegebene Umwandlungsschema wird durch die experimentellen Ergebnisse über die $\alpha$-Strahlung ron Radioactinium gestutzt. Die neuen Isotopen der Actiniumzerfallsprodukte sind vorerst noch hypothetisch. Thre Existenz wird nur durch besondere Experimentalversuche festgestellt werden können.

Während die Umwandlungsschematen 1., 2. und 3. für das Atomgewicht des Endproduktes der Actiniumfamilie einen um vier Einheiten niedrigeren Wert (202 statt 206) rerlangen, hat unser neues Zerfallsschema den Vorteil, daB das Atomgewicht des Endproduktes ungeändert bleibt.

Kristiania, den 7. Augusi 1921.

Bei der Redaktion eingegangen am 22. August 1921. 